\title{
Spatial Behavior of a Coupled System of Wave-Plate Type
}

\author{
Gusheng Tang, ${ }^{1}$ Yan Liu, ${ }^{2}$ and Wenhui Liao' \\ ${ }^{1}$ Department of Mathematics, Hunan University of Science and Technology, Hunan, Xiangtan 411201, China \\ ${ }^{2}$ Department of Applied Mathematics, Guangdong University of Finance, Guangzhou 510521, China
}

Correspondence should be addressed to Yan Liu; liuyan99021324@tom.com

Received 9 February 2014; Revised 21 May 2014; Accepted 6 June 2014; Published 23 June 2014

Academic Editor: Stanislaw Migorski

Copyright (C) 2014 Gusheng Tang et al. This is an open access article distributed under the Creative Commons Attribution License, which permits unrestricted use, distribution, and reproduction in any medium, provided the original work is properly cited.

\begin{abstract}
The spatial behavior of a coupled system of wave-plate type is studied. We get the alternative results of Phragmén-Lindelöf type in terms of an area measure of the amplitude in question based on a first-order differential inequality. We also get the spatial decay estimates based on a second-order differential inequality.
\end{abstract}

\section{Introduction and Preliminaries}

Principle of Saint-Venant type used to be one of the most popular subjects of applied mathematics and mechanics in the 1960-1980s. A great number of investigation results have expanded enormously the classical Saint-Venant principle. For a review of recent work on Saint-Venant's principle, one may refer to the review papers by Horgan $[1,2]$ and Horgan and Knowles [3]. A common feature of Saint-Venant-type theorems is to establish the exponential decay estimates of energy with axial distance from the near end of a semi-infinite strip or cylinder. Furthermore, all papers need to impose a priori decay assumption at infinity. In recent years, the investigations of Saint-Venant principle are mainly on the studies of the Phragmén-Lindelöf alternative principle. The classical Phragmén-Lindelöf theorem has also extensively extended by numerous investigation results. It shows that Phragmén-Lindelöf alternative principle is of theoretical and applied significance in physics mechanics and other applied sciences. The spatial behaviour for several types of partial differential equations and systems has been the subject of extensive investigating in the literature for close to a century and a half. These studies were motivated by a desire to formulate Saint-Venant and Phragmén-Lindelöf-type principles. Roughly speaking, these results assert that the solution of the problem decays exponentially with distance from the boundary. For a more complete view for the spatial behavior, one could refer to [4-8].
A spatial behavior study for the transient heat conduction was first given by Edelstein [9]. Since then, many works have been devoted to study the spatial behaviour of parabolic equations (see $[10,11])$. Little attention has been paid to the study of the hyperbolic equations. Horgan $[1,2]$ and Horgan and Knowles [3] point out the paucity of Saint-Venanttype results for hyperbolic system of the kind describing elastic wave propagation. The first contribution in hyperbolic equations concerning the Saint-Venant's principle may be due to Knops and Payne [12]. In recent years, the study of end decay effects for hyperbolic and quasihyperbolic equations has grown in a relevant form. It is worth recalling some recent contributions concerning the spatial behavior of viscoelasticity equations; one could refer to [13-16].

The biharmonic equation has important applications in the study of the applied mathematics and mechanics. Many studies and various methods have been proposed for researching the spatial behaviour for the solutions of the biharmonic equations in a semi-infinite strip in $R^{2}$. We mention the studies by Knowles [17, 18], Flavin [19], Flavin and Knops [20], Horgan [21], Payne and Schaefer [22], and Varlamov [23]. Additional references may be found in the review papers [1-3]. We note that some time-dependent problems concerning the biharmonic operator are considered in the literature; we mention the papers by Lin [10] and Knops and Lupoli [24] in connection with the spatial behaviour of solutions for a fourth-order transformed problem associated with the slow flow of an incompressible viscous fluid along 
a semi-infinite strip. In recent years, Song in his paper $[25,26]$ improved the result obtained by Lin in [10] for the timedependent Stokes flow.

In this paper, the spatial behavior of solutions of the fourth-order hyperbolic equations is studied. Growth and decay estimates are established associating some appropriate cross-sectional line and area integral measures. The method of the proof is based on a first-order differential inequality leading to an alternative of Phragmén-Lindelöf type in terms of an area measure of the amplitude in question. We also get the spatial decay estimates based on a second-order differential inequality, and we also indicate how to bound the total energy.

In the present paper, the comma is used to indicate partial differentiation and the differentiation with respect to the direction $x_{k}$ is denoted as, $k$; thus $u_{, \alpha}$ denotes $\partial u / \partial x_{\alpha}$, and $u_{, t}$ denotes $\partial u / \partial t$. The usual summation convection is employed with repeated Greek subscripts $\alpha$ summed from 1 to 2 . Hence, $u_{\alpha, \alpha}=\sum_{\alpha=1}^{2}\left(\partial u_{\alpha} / \partial x_{\alpha}\right)$

\section{Formulation}

We consider the problem on an unbounded region $\Omega_{0}$ defined by

$$
\Omega_{0}:=\left\{\left(x_{1}, x_{2}\right) \mid x_{1}>0,0<x_{2}<h\right\}
$$

where $h$ is a fixed constant, and we introduce the notation

$$
L_{z}=\left\{\left(x_{1}, x_{2}\right) \mid x_{1}=z \geq 0,0 \leq x_{2} \leq h\right\}
$$

We consider the problem in the time interval $[0, T]$, where $T$ is a fixed positive constant.

In [27], the authors studied the analytic property and the exponential stability of the $C_{0}$-semigroup associated with the following coupled system of wave-plate type with thermal effect:

$$
\begin{gathered}
\rho_{1} u_{, t t}-\Delta u-\mu \Delta u_{, t}+a \Delta v=0, \\
\rho_{2} v_{, t t}+\gamma \Delta^{2} v+a \Delta u+m \Delta \theta=0, \\
\tau \theta_{, t}-k \Delta \theta-m \Delta v_{, t}=0 .
\end{gathered}
$$

The above model can be used to describe the evolution of a system consisting of an elastic membrane and an elastic plate, subject to an elastic force that attracts the membrane to the plate with coefficient $a$, subject to a thermal effect (see [28]). Here $u$ and $v$ represent the vertical deflections of the membrane and of the plate, respectively. $\theta$ denotes the difference of temperature. The coefficients $\rho_{1}, \rho_{2}, \mu, a, \gamma, m, \tau$, and $k$ are nonnegative constants.

Their main result obtained in [27] was that the semigroup associated with the system is analytic. This is to say that the exponential stability of the associated energy and also the so-called spectrum determined growth property (SDG property) of the corresponding semigroup.
In the present paper, we consider the system (4)-(6) with the case $\tau=0$. The equations are

$$
\begin{gathered}
\rho_{1} u_{, t t}-\Delta u-\mu \Delta u_{, t}+a \Delta v=0, \\
\rho_{2} v_{, t t}+\gamma \Delta^{2} v+a \Delta u-\frac{m^{2}}{k} \Delta v_{, t}=0,
\end{gathered}
$$

and the initial boundary conditions are

$$
\begin{gathered}
u\left(x_{1}, 0, t\right)=v\left(x_{1}, 0, t\right)=v_{, 2}\left(x_{1}, 0, t\right)=0 \quad x_{1}>0, t>0, \\
u\left(x_{1}, h, t\right)=v\left(x_{1}, h, t\right)=v_{, 2}\left(x_{1}, h, t\right)=0 \quad x_{1}>0, t>0, \\
u\left(0, x_{2}, t\right)=g_{1}\left(x_{2}, t\right) \quad 0 \leq x_{2} \leq h, t>0, \\
v\left(0, x_{2}, t\right)=g_{2}\left(x_{2}, t\right) \quad 0 \leq x_{2} \leq h, t>0, \\
v_{, 1}\left(0, x_{2}, t\right)=g_{3}\left(x_{2}, t\right) \quad 0 \leq x_{2} \leq h, t>0, \\
u\left(x_{1}, x_{2}, 0\right)=v\left(x_{1}, x_{2}, 0\right)=u_{, t}\left(x_{1}, x_{2}, 0\right) \\
=v_{, t}\left(x_{1}, x_{2}, 0\right)=0 \\
0 \leq x_{2} \leq h, x_{1}>0 .
\end{gathered}
$$

Here $\Delta$ is the harmonic operator, and $\Delta^{2}$ is the biharmonic operator.

In this paper, we consider the classical solutions to the problem (4)-(6). $g_{i}\left(x_{2}, t\right) i=1,2,3$ are prescribed functions satisfying the compatibility:

$$
\begin{gathered}
g_{1}(0, t)=g_{1}(h, t)=g_{1,2}(0, t)=g_{1,2}(h, t)=0, \\
g_{2}(0, t)=g_{2}(h, t)=g_{2,2}(0, t)=g_{2,2}(h, t)=0, \\
g_{3}(0, t)=g_{3}(h, t)=g_{3,2}(0, t)=g_{3,2}(h, t)=0, \\
g_{1}\left(x_{2}, 0\right)=g_{2}\left(x_{2}, 0\right)=g_{3}\left(x_{2}, 0\right)=0 .
\end{gathered}
$$

We will use the Poincare inequality; we recall that the estimate

$$
\int_{0}^{h} u^{2} d \xi \leq \frac{h^{2}}{\pi^{2}} \int_{0}^{h}\left(\frac{d u}{d \xi}\right)^{2} d \xi
$$

is satisfied for smooth functions $u(\xi)$ such that $u(0)=0$ (see [2]). 
Abstract and Applied Analysis

3

On using the divergence theorem and the initial-boundary conditions (4)-(6), it leads to

$$
\begin{aligned}
0= & \int_{0}^{t} \int_{z_{0}}^{z} \int_{L_{\xi}} u_{, \eta}\left(\rho_{1} u_{, \eta \eta}-u_{, \alpha \alpha}-\mu u_{, \alpha \alpha \eta}+a v_{, \alpha \alpha}\right) d A d \eta \\
= & \left.\frac{\rho_{1}}{2} \int_{z_{0}}^{z} \int_{L_{\xi}} u_{, \eta}^{2} d A\right|_{\eta=t}+\left.\frac{1}{2} \int_{z_{0}}^{z} \int_{L_{\xi}} u_{, \alpha} u_{, \alpha} d A\right|_{\eta=t} \\
& -\int_{0}^{t} \int_{L_{z}} u_{, \eta} u_{, 1} d x_{2} d \eta+\int_{0}^{t} \int_{L_{z_{0}}} u_{, \eta} u_{, 1} d x_{2} d \eta \\
& +\mu \int_{0}^{t} \int_{z_{0}}^{z} \int_{L_{\xi}} u_{, \alpha \eta} u_{, \alpha \eta} d A d \eta-\mu \int_{0}^{t} \int_{L_{z}} u_{, \eta} u_{, 1 \eta} d x_{2} d \eta \\
& +\mu \int_{0}^{t} \int_{L_{z_{0}}} u_{, \eta} u_{, 1 \eta} d x_{2} d \eta \\
& +a \int_{0}^{t} \int_{z_{0}}^{z} \int_{L_{\xi}} u_{, \eta} v_{, \alpha \alpha} d A d \eta .
\end{aligned}
$$

We can also get

$$
\begin{aligned}
0= & \int_{0}^{t} \int_{z_{0}}^{z} \int_{L_{\xi}} v_{, \eta}\left(\rho_{2} v_{, \eta \eta}+\gamma v_{, \alpha \alpha \beta \beta}+a u_{, \alpha \alpha}-\frac{m^{2}}{k} v_{, \alpha \alpha \eta}\right) d A d \eta \\
= & \left.\frac{\rho_{2}}{2} \int_{z_{0}}^{z} \int_{L_{\xi}} v_{, \eta}^{2} d A\right|_{\eta=t}+\left.\frac{\gamma}{2} \int_{z_{0}}^{z} \int_{L_{\xi}} v_{, \alpha \beta} v_{, \alpha \beta} d A\right|_{\eta=t} \\
& -\gamma \int_{0}^{t} \int_{L_{z}} v_{, \alpha \eta} v_{, 1 \alpha} d x_{2} d \eta+\gamma \int_{0}^{t} \int_{L_{z_{0}}} v_{, \alpha \eta} v_{, 1 \alpha} d x_{2} d \eta \\
& +\gamma \int_{0}^{t} \int_{L_{z}} v_{, \eta} v_{, 1 \beta \beta} d x_{2} d \eta-\gamma \int_{0}^{t} \int_{L_{z_{0}}} v_{, \eta} v_{, 1 \beta \beta} d x_{2} d \eta \\
& +a \int_{0}^{t} \int_{z_{0}}^{z} \int_{L_{\xi}} v_{, \eta} u_{, \alpha \alpha} d A d \eta \\
& +\frac{m^{2}}{k} \int_{0}^{t} \int_{z_{0}}^{z} \int_{L_{\xi}} v_{, \alpha \eta} v_{, \alpha \eta} d A d \eta-\frac{m^{2}}{k} \int_{0}^{t} \int_{L_{z}} v_{, \eta} v_{, 1 \eta} d x_{2} d \eta \\
& +\frac{m^{2}}{k} \int_{0}^{t} \int_{L_{z_{0}}} v_{, \eta} v_{, 1 \eta} d x_{2} d \eta .
\end{aligned}
$$

Integrating by parts, we can get

$$
\begin{aligned}
& \int_{0}^{t} \int_{z_{0}}^{z} \int_{L_{\xi}} u_{, \eta} v_{, \alpha \alpha} d A d \eta \\
& =-\int_{0}^{t} \int_{z_{0}}^{z} \int_{L_{\xi}} u_{, \alpha \eta} v_{, \alpha} d A d \eta \\
& \quad+\int_{0}^{t} \int_{L_{z}} u_{, \eta} v_{, 1} d x_{2} d \eta-\int_{0}^{t} \int_{L_{z_{0}}} u_{, \eta} v_{, 1} d x_{2} d \eta \\
& =\int_{0}^{t} \int_{z_{0}}^{z} \int_{L_{\xi}} u_{, \alpha} v_{, \alpha \eta} d A d \eta-\left.\int_{z_{0}}^{z} \int_{L_{\xi}} u_{, \alpha} v_{, \alpha} d A\right|_{\eta=t}
\end{aligned}
$$

$$
\begin{aligned}
& +\int_{0}^{t} \int_{L_{z}} u_{, \eta} v_{, 1} d x_{2} d \eta-\int_{0}^{t} \int_{L_{z_{0}}} u_{, \eta} v_{, 1} d x_{2} d \eta, \\
& \int_{0}^{t} \int_{z_{0}}^{z} \int_{L_{\xi}} v_{, \eta} u_{, \alpha \alpha} d A d \eta \\
& =-\int_{0}^{t} \int_{z_{0}}^{z} \int_{L_{\xi}} v_{, \alpha \eta} u_{, \alpha} d A d \eta \\
& \quad+\int_{0}^{t} \int_{L_{z}} v_{, \eta} u_{, 1} d x_{2} d \eta-\int_{0}^{t} \int_{L_{z_{0}}} v_{, \eta} u_{, 1} d x_{2} d \eta .
\end{aligned}
$$

Combining the above computations, we define a function

$$
\begin{aligned}
& \phi_{1}(z, t)=\int_{0}^{t} \int_{L_{z}} u_{, \eta} u_{, 1} d x_{2} d \eta+\mu \int_{0}^{t} \int_{L_{z}} u_{, \eta} u_{, 1 \eta} d x_{2} d \eta \\
& -a \int_{0}^{t} \int_{L_{z}} u_{, \eta} v_{, 1} d x_{2} d \eta+\gamma \int_{0}^{t} \int_{L_{z}} v_{, \alpha \eta} v_{, 1 \alpha} d x_{2} d \eta \\
& -\gamma \int_{0}^{t} \int_{L_{z}} v_{, \eta} v_{, 1 \beta \beta} d x_{2} d \eta+\frac{m^{2}}{k} \int_{0}^{t} \int_{L_{z}} v_{, \eta} v_{, 1 \eta} d x_{2} d \eta \\
& -a \int_{0}^{t} \int_{L_{z}} v_{, \eta} u_{, 1} d x_{2} d \eta \\
& =\left.\frac{\rho_{1}}{2} \int_{z_{0}}^{z} \int_{L_{\xi}} u_{, \eta}^{2} d A\right|_{\eta=t}+\left.\frac{1}{2} \int_{z_{0}}^{z} \int_{L_{\xi}} u_{, \alpha} u_{, \alpha} d A\right|_{\eta=t} \\
& +\mu \int_{0}^{t} \int_{z_{0}}^{z} \int_{L_{\xi}} u_{, \alpha \eta} u_{, \alpha \eta} d A d \eta+\left.\frac{\rho_{2}}{2} \int_{z_{0}}^{z} \int_{L_{\xi}} v_{, \eta}^{2} d A\right|_{\eta=t} \\
& +\left.\frac{\gamma}{2} \int_{z_{0}}^{z} \int_{L_{\xi}} v_{, \alpha \beta} v_{, \alpha \beta} d A\right|_{\eta=t} \\
& +\frac{m^{2}}{k} \int_{0}^{t} \int_{z_{0}}^{z} \int_{L_{\xi}} v_{, \alpha \eta} v_{, \alpha \eta} d A d \eta \\
& -\left.a \int_{z_{0}}^{z} \int_{L_{\xi}} u_{, \alpha} v_{, \alpha} d A\right|_{\eta=t}+\phi_{1}\left(z_{0}, t\right) .
\end{aligned}
$$

We can also get

$$
\begin{array}{r}
\int_{0}^{t} \int_{z_{0}}^{z} \int_{L_{\xi}} v_{, 11}\left(\rho_{2} v_{, \eta \eta}+\gamma v_{, \alpha \alpha \beta \beta}\right. \\
\left.+a u_{, \alpha \alpha}-\frac{m^{2}}{k} v_{, \alpha \alpha \eta}\right) d A d \eta=0 .
\end{array}
$$


4

Abstract and Applied Analysis

We have

$$
\begin{aligned}
& \int_{0}^{t} \int_{z_{0}}^{z} \int_{L_{\xi}} \rho_{2} v_{, 11} v_{, \eta \eta} d A d \eta \\
& =\int_{0}^{t} \int_{z_{0}}^{z} \int_{L_{\xi}} \rho_{2} v_{, 1 \eta} v_{, 1 \eta} d A d \eta \\
& -\int_{0}^{t} \int_{L_{z}} \rho_{2} v_{, 1 \eta} v_{, \eta} d x_{2} d \eta \\
& +\int_{0}^{t} \int_{L_{z_{0}}} \rho_{2} v_{, 1 \eta} v_{, \eta} d x_{2} d \eta+\left.\int_{z_{0}}^{z} \int_{L_{\xi}} \rho_{2} v_{, 11} v_{, \eta} d A\right|_{\eta=t}, \\
& \int_{0}^{t} \int_{z_{0}}^{z} \int_{L_{\xi}} v_{, 11}\left(\gamma v_{, \alpha \alpha \beta \beta}-\frac{m^{2}}{k} v_{, \alpha \alpha \eta}\right) d A d \eta \\
& =-\gamma \int_{0}^{t} \int_{z_{0}}^{z} \int_{L_{\xi}} v_{, 1 \alpha \beta} v_{, 1 \alpha \beta} d A d \eta \\
& -\left.\frac{m^{2}}{2 k} \int_{z_{0}}^{z} \int_{L_{\xi}} v_{, 1 \alpha} v_{, 1 \alpha} d A\right|_{\eta=t} \\
& +\int_{0}^{t} \int_{L_{z}} \gamma v_{, \alpha 2} v_{, 1 \alpha 2} d x_{2} d \eta+\frac{m^{2}}{k} \int_{0}^{t} \int_{L_{z}} v_{, 12} v_{, 2 \eta} d x_{2} d \eta \\
& +\gamma \int_{0}^{t} \int_{L_{z}} v_{, 11} v_{, 1 \beta \beta} d x_{2} d \eta-\int_{0}^{t} \int_{L_{z_{0}}} \gamma v_{, \alpha 2} v_{, 1 \alpha 2} d x_{2} d \eta \\
& -\frac{m^{2}}{k} \int_{0}^{t} \int_{L_{z_{0}}} v_{, 12} v_{, 2 \eta} d x_{2} d \eta-\gamma \int_{0}^{t} \int_{L_{z_{0}}} v_{, 11} v_{, 1 \beta \beta} d x_{2} d \eta, \\
& \int_{0}^{t} \int_{z_{0}}^{z} \int_{L_{\xi}} a v_{, 11} u_{, \alpha \alpha} d A d \eta \\
& =-\int_{0}^{t} \int_{z_{0}}^{z} \int_{L_{\xi}} a v_{, 11 \alpha} u_{, \alpha} d A d \eta+\int_{0}^{t} \int_{L_{z}} a v_{, 11} u_{, 1} d x_{2} d \eta \\
& -\int_{0}^{t} \int_{L_{z_{0}}} a v_{, 11} u_{, 1} d x_{2} d \eta
\end{aligned}
$$

We now define another function:

$$
\begin{aligned}
\phi_{2}(z, t)= & -\int_{0}^{t} \int_{L_{z}} \rho_{2} v_{, 1 \eta} v_{, \eta} d x_{2} d \eta+\int_{0}^{t} \int_{L_{z}} \gamma v_{, \alpha 2} v_{, 1 \alpha 2} d x_{2} d \eta \\
& +\frac{m^{2}}{k} \int_{0}^{t} \int_{L_{z}} v_{, 12} v_{, 2 \eta} d x_{2} d \eta \\
& +\gamma \int_{0}^{t} \int_{L_{z}} v_{, 11} v_{, 1 \beta \beta} d x_{2} d \eta+a \int_{0}^{t} \int_{L_{z}} v_{, 11} u_{, 1} d x_{2} d \eta \\
= & -\int_{0}^{t} \int_{z_{0}}^{z} \int_{L_{\xi}} \rho_{2} v_{, 1 \eta} v_{, 1 \eta} d A d \eta \\
& -\left.\int_{z_{0}}^{z} \int_{L_{\xi}} \rho_{2} v_{, 11} v_{, \eta} d A\right|_{\eta=t}
\end{aligned}
$$

$$
\begin{aligned}
& +\gamma \int_{0}^{t} \int_{z_{0}}^{z} \int_{L_{\xi}} v_{, 1 \alpha \beta} v_{, 1 \alpha \beta} d A d \eta \\
& +\left.\frac{m^{2}}{2 k} \int_{z_{0}}^{z} \int_{L_{\xi}} v_{, 1 \alpha} v_{, 1 \alpha} d A\right|_{\eta=t} \\
& +\int_{0}^{t} \int_{z_{0}}^{z} \int_{L_{\xi}} a v_{, 11 \alpha} u_{, \alpha} d A d \eta+\phi_{2}\left(z_{0}, t\right) .
\end{aligned}
$$

We define a new function:

$$
\begin{aligned}
& \Phi(z, t)=\phi_{1}(z, t)+\lambda \phi_{2}(z, t) \\
& =\int_{0}^{t} \int_{L_{z}} u_{, \eta} u_{, 1} d x_{2} d \eta \\
& +\mu \int_{0}^{t} \int_{L_{z}} u_{, \eta} u_{, 1 \eta} d x_{2} d \eta \\
& -a \int_{0}^{t} \int_{L_{z}} u_{, \eta} v_{, 1} d x_{2} d \eta \\
& +\gamma \int_{0}^{t} \int_{L_{z}} v_{, \alpha \eta} v_{, 1 \alpha} d x_{2} d \eta \\
& +\gamma \int_{0}^{t} \int_{L_{z}} v_{, \eta} v_{, 1 \beta \beta} d x_{2} d \eta \\
& +\frac{m^{2}}{k} \int_{0}^{t} \int_{L_{z}} v_{, \eta} v_{, 1 \eta} d x_{2} d \eta \\
& -a \int_{0}^{t} \int_{L_{z}} u_{, \eta} u_{, 1} d x_{2} d \eta \\
& -\lambda \int_{0}^{t} \int_{L_{z}} \rho_{2} v_{, 1 \eta} v_{, \eta} d x_{2} d \eta \\
& +\lambda \int_{0}^{t} \int_{L_{z}} \gamma v_{, \alpha 2} v_{, 1 \alpha 2} d x_{2} d \eta \\
& +\lambda \frac{m^{2}}{k} \int_{0}^{t} \int_{L_{z}} v_{, 12} v_{, 2 \eta} d x_{2} d \eta \\
& +\lambda \gamma \int_{0}^{t} \int_{L_{z}} v_{, 11} v_{, 1 \beta \beta} d x_{2} d \eta \\
& +a \lambda \int_{0}^{t} \int_{L_{z}} v_{, 11} u_{, 1} d x_{2} d \eta \\
& =\left.\frac{\rho_{1}}{2} \int_{z_{0}}^{z} \int_{L_{\xi}} u_{, \eta}^{2} d A\right|_{\eta=t} \\
& +\left.\frac{1}{2} \int_{z_{0}}^{z} \int_{L_{\xi}} u_{, \alpha} u_{, \alpha} d A\right|_{\eta=t} \\
& +\mu \int_{0}^{t} \int_{z_{0}}^{z} \int_{L_{\xi}} u_{, \alpha \eta} u_{, \alpha \eta} d A d \eta
\end{aligned}
$$


Abstract and Applied Analysis

5

$$
\begin{aligned}
& +\left.\frac{\rho_{2}}{2} \int_{z_{0}}^{z} \int_{L_{\xi}} v_{, \eta}^{2} d A\right|_{\eta=t} \\
& +\left.\frac{\gamma}{2} \int_{z_{0}}^{z} \int_{L_{\xi}} v_{, \alpha \beta} v_{, \alpha \beta} d A\right|_{\eta=t} \\
& +\frac{m^{2}}{k} \int_{0}^{t} \int_{z_{0}}^{z} \int_{L_{\xi}} v_{, \alpha \eta} v_{, \alpha \eta} d A d \eta \\
& -\left.a \int_{z_{0}}^{z} \int_{L_{\xi}}^{u} u_{, \alpha} v_{, \alpha} d A\right|_{\eta=t} \\
& -\lambda \int_{0}^{t} \int_{z_{0}}^{z} \int_{L_{\xi}} \rho_{2} v_{, 1 \eta} v_{, 1 \eta} d A d \eta \\
& +\lambda \int_{0}^{z} \int_{z_{0}}^{z} \int_{L_{\xi}}^{z} a v_{, 11 \alpha} u_{, \alpha} d A d \eta+\Phi\left(z_{0}, t\right) \\
& +\left.\left.\lambda \gamma \int_{0}^{t} \int_{z_{0}}^{z} \int_{L_{\xi}, 11} v_{, \eta} v_{, 1 \alpha \beta} d A\right|_{\eta=t} ^{z} \int_{z_{0}}^{z} \int_{L_{\xi}} v_{, 1 \alpha} v_{, 1 \alpha} d A\right|_{\eta=t} d \eta
\end{aligned}
$$

where $\lambda$ is a positive constant to be determined later.

From (16), we can easily get

$$
\begin{aligned}
\frac{\partial \Phi(z, t)}{\partial z}= & \left.\frac{\rho_{1}}{2} \int_{L_{z}} u_{, \eta}^{2} d x_{2}\right|_{\eta=t}+\left.\frac{1}{2} \int_{L_{z}} u_{, \alpha} u_{, \alpha} d x_{2}\right|_{\eta=t} \\
& +\mu \int_{0}^{t} \int_{L_{z}} u_{, \alpha \eta} u_{, \alpha \eta} d x_{2} d \eta \\
& +\left.\frac{\rho_{2}}{2} \int_{L_{z}} v_{, \eta}^{2} d x_{2}\right|_{\eta=t}+\left.\frac{\gamma}{2} \int_{L_{z}} v_{, \alpha \beta} v_{, \alpha \beta} d x_{2}\right|_{\eta=t} \\
& +\frac{m^{2}}{k} \int_{0}^{t} \int_{L_{z}} v_{, \alpha \eta} v_{, \alpha \eta} d x_{2} d \eta \\
& -\left.a \int_{L_{z}} u_{, \alpha} v_{, \alpha} d x_{2}\right|_{\eta=t}-\lambda \int_{0}^{t} \int_{L_{z}} \rho_{2} v_{, 1 \eta} v_{, 1 \eta} d x_{2} d \eta \\
& -\left.\lambda \int_{L_{z}} \rho_{2} v_{, 11} v_{, \eta} d x_{2}\right|_{\eta=t} ^{t} \\
& +\lambda \gamma \int_{0}^{t} \int_{L_{z}} v_{, 1 \alpha \beta} v_{, 1 \alpha \beta} d x_{2} d \eta
\end{aligned}
$$

$$
\begin{aligned}
& +\left.\lambda \frac{m^{2}}{2 k} \int_{L_{z}} v_{, 1 \alpha} v_{, 1 \alpha} d x_{2}\right|_{\eta=t} \\
& +\lambda \int_{0}^{t} \int_{L_{z}} a v_{, 11 \alpha} u_{, \alpha} d x_{2} d \eta
\end{aligned}
$$

Using the Cauchy's inequality and the inequality (8), we have

$$
\begin{aligned}
& \left|\lambda \int_{0}^{t} \int_{L_{z}} a v_{, 11 \alpha} u_{, \alpha} d x_{2} d \eta\right| \\
& \leq \frac{\lambda \gamma}{4} \int_{0}^{t} \int_{L_{z}} v_{, 11 \alpha} v_{, 11 \alpha} d x_{2} d \eta \\
& +\frac{\lambda a^{2} t^{2}}{\pi^{2} \gamma} \int_{0}^{t} \int_{L_{z}} u_{, \alpha \eta} u_{, \alpha \eta} d x_{2} d \eta, \\
& \left|a \int_{L_{z}} u_{, \alpha} v_{, \alpha} d x_{2}\right|_{\eta=t} \mid \\
& \leq\left.\frac{1}{4} \int_{L_{z}} u_{, \alpha} u_{, \alpha} d x_{2}\right|_{\eta=t} \\
& +\left.\frac{a^{2} h^{2}}{\pi^{2}} \int_{L_{z}} v_{, \alpha \beta} v_{, \alpha \beta} d x_{2}\right|_{\eta=t}, \\
& \left|\rho_{2} \lambda \int_{L_{z}} v_{, 11} v_{, \eta} d x_{2}\right|_{\eta=t} \mid \\
& \leq\left.\frac{\gamma}{8} \int_{L_{z}} v_{, 11}^{2} d x_{2}\right|_{\eta=t} \\
& +\left.\frac{2 \rho_{2}^{2} \lambda^{2}}{c} \int_{L_{z}} v_{, \eta}^{2} d x_{2}\right|_{\eta=t} .
\end{aligned}
$$

For $0 \leq t \leq T$, we can choose $a$ and $\lambda$ satisfying

$$
\begin{array}{ll}
\rho_{2} \lambda \leq \frac{m^{2}}{2 k}, & \frac{\lambda a^{2} t^{2}}{\pi^{2} \gamma} \leq \frac{\mu}{2}, \\
\frac{a^{2} h^{2}}{\pi^{2}} \leq \frac{\gamma}{8}, & \frac{\rho_{2} \lambda^{2}}{c} \leq \frac{1}{8} .
\end{array}
$$

Combining the above discussions, we obtain

$$
\begin{aligned}
\frac{\partial \Phi(z, t)}{\partial z} \geq & \left.\frac{\rho_{1}}{4} \int_{L_{z}} u_{, \eta}^{2} d x_{2}\right|_{\eta=t}+\left.\frac{1}{4} \int_{L_{z}} u_{, \alpha} u_{, \alpha} d x_{2}\right|_{\eta=t} \\
& +\frac{\mu}{2} \int_{0}^{t} \int_{L_{z}} u_{, \alpha \eta} u_{, \alpha \eta} d x_{2} d \eta \\
& +\left.\frac{\rho_{2}}{4} \int_{L_{z}} v_{, \eta}^{2} d x_{2}\right|_{\eta=t}+\left.\frac{\gamma}{4} \int_{L_{z}} v_{, \alpha \beta} v_{, \alpha \beta} d x_{2}\right|_{\eta=t}
\end{aligned}
$$




$$
\begin{aligned}
& +\frac{m^{2}}{2 k} \int_{0}^{t} \int_{L_{z}} v_{, \alpha \eta} v_{, \alpha \eta} d x_{2} d \eta \\
& +\frac{\lambda \gamma}{2} \int_{0}^{t} \int_{L_{z}} v_{, 1 \alpha \beta} v_{, 1 \alpha \beta} d x_{2} d \eta \\
& +\left.\lambda \frac{m^{2}}{4 k} \int_{L_{z}} v_{, 1 \alpha} v_{, 1 \alpha} d x_{2}\right|_{\eta=t} .
\end{aligned}
$$

\section{Phragmén-Lindelöf Alternative Results}

In this part, we will derive the Phragmén-Lindelöf alternative results based on a first-order differential inequality.

From the definition of $\Phi(z, t)$ in (16), we want to give bounds for items in $\Phi(z, t)$. Using the Schwarz's inequality and the inequality (8), we can easily obtain

$$
\begin{aligned}
& \left|\int_{0}^{t} \int_{L_{z}} u_{, \eta} u_{, 1} d x_{2} d \eta\right| \leq \frac{h^{2}}{2 \pi^{2}} \int_{0}^{t} \int_{L_{z}} u_{, 2 \eta}^{2} d x_{2} d \eta \\
& +\frac{t^{2}}{2 \pi^{2}} \int_{0}^{t} \int_{L_{z}} u_{, 1 \eta}^{2} d x_{2} d \eta \\
& \left|\mu \int_{0}^{t} \int_{L_{z}} u_{, \eta} u_{, 1 \eta} d x_{2} d \eta\right| \leq \frac{\mu h^{2}}{2 \pi^{2}} \int_{0}^{t} \int_{L_{z}} u_{, 2 \eta}^{2} d x_{2} d \eta \\
& +\frac{\mu}{2} \int_{0}^{t} \int_{L_{z}} u_{, 1 \eta}^{2} d x_{2} d \eta \\
& \left|a \int_{0}^{t} \int_{L_{z}} u_{, \eta} v_{, 1} d x_{2} d \eta\right| \leq \frac{a h^{2}}{2 \pi^{2}} \int_{0}^{t} \int_{L_{z}} u_{, 2 \eta}^{2} d x_{2} d \eta \\
& +\frac{a t^{2}}{2 \pi^{2}} \int_{0}^{t} \int_{L_{z}} v_{, 1 \eta}^{2} d x_{2} d \eta \\
& \left|\gamma \int_{0}^{t} \int_{L_{z}} v_{, \eta} v_{, 1 \beta \beta} d x_{2} d \eta\right| \leq \frac{\gamma h^{2}}{2 \pi^{2}} \int_{0}^{t} \int_{L_{z}} v_{, 2 \eta}^{2} d x_{2} d \eta \\
& +\frac{\gamma}{2} \int_{0}^{t} \int_{L_{z}} v_{, 1 \beta \beta}^{2} d x_{2} d \eta \\
& \left|\gamma \int_{0}^{t} \int_{L_{z}} v_{, \alpha \eta} v_{, 1 \alpha} d x_{2} d \eta\right| \leq \frac{\gamma}{2} \int_{0}^{t} \int_{L_{z}} v_{, \alpha \eta} v_{, \alpha \eta} d x_{2} d \eta \\
& +\frac{\gamma h^{2}}{2 \pi^{2}} \int_{0}^{t} \int_{L_{z}} v_{, 1 \beta 2} v_{, 1 \beta 2} d x_{2} d \eta \\
& \left|\frac{m^{2}}{k} \int_{0}^{t} \int_{L_{z}} v_{, \eta} v_{, 1 \eta} d x_{2} d \eta\right| \leq \frac{m^{2} h^{2}}{2 k \pi^{2}} \int_{0}^{t} \int_{L_{z}} v_{, 2 \eta}^{2} d x_{2} d \eta \\
& +\frac{m^{2}}{2 k} \int_{0}^{t} \int_{L_{z}} v_{, 1 \eta}^{2} d x_{2} d \eta \\
& \left|a \int_{0}^{t} \int_{L_{z}} u_{, \eta} u_{, 1} d x_{2} d \eta\right| \leq \frac{a h^{2}}{2 \pi^{2}} \int_{0}^{t} \int_{L_{z}} u_{, 2 \eta}^{2} d x_{2} d \eta
\end{aligned}
$$

$$
\begin{aligned}
& +\frac{a t^{2}}{2 \pi^{2}} \int_{0}^{t} \int_{L_{z}} u_{, 1 \eta}^{2} d x_{2} d \eta, \\
\left|\lambda \rho_{2} \int_{0}^{t} \int_{L_{z}} v_{, 1 \eta} v_{, \eta} d x_{2} d \eta\right| \leq & \frac{\lambda \rho_{2}}{2 \pi^{2}} \int_{0}^{t} \int_{L_{z}} v_{, 1 \eta}^{2} d x_{2} d \eta \\
& +\frac{\lambda \rho_{2} h^{2}}{2 \pi^{2}} \int_{0}^{t} \int_{L_{z}} v_{, 2 \eta}^{2} d x_{2} d \eta,
\end{aligned}
$$$$
\left|\lambda \gamma \int_{0}^{t} \int_{L_{z}} v_{, \alpha 2} v_{, 1 \alpha 2} d x_{2} d \eta\right| \leq \frac{\lambda \gamma}{2} \int_{0}^{t} \int_{L_{z}} v_{, \alpha \beta} v_{, \alpha \beta} d x_{2} d \eta
$$$$
+\frac{\lambda \gamma}{2} \int_{0}^{t} \int_{L_{z}} v_{, 1 \alpha \beta} v_{, 1 \alpha \beta} d x_{2} d \eta
$$$$
\left|\frac{\lambda m^{2}}{k} \int_{0}^{t} \int_{L_{z}} v_{, 12} v_{, 2 \eta} d x_{2} d \eta\right| \leq \frac{\lambda h^{2} m^{2}}{2 \pi^{2} k} \int_{0}^{t} \int_{L_{z}} v_{, 122}^{2} d x_{2} d \eta
$$$$
+\frac{\lambda m^{2}}{2 k} \int_{0}^{t} \int_{L_{z}} v_{, 2 \eta}^{2} d x_{2} d \eta
$$$$
\left|\lambda \gamma \int_{0}^{t} \int_{L_{z}} v_{, 11} v_{, 1 \beta \beta} d x_{2} d \eta\right| \leq \frac{\lambda \gamma h^{2}}{2 \pi^{2}} \int_{0}^{t} \int_{L_{z}} v_{, 112}^{2} d x_{2} d \eta
$$$$
+\frac{\lambda \gamma}{2} \int_{0}^{t} \int_{L_{z}} v_{, 1 \beta \beta}^{2} d x_{2} d \eta
$$$$
\left|a \lambda \int_{0}^{t} \int_{L_{z}} v_{, 11} u_{, 1} d x_{2} d \eta\right| \leq \frac{a \lambda h^{2}}{2 \pi^{2}} \int_{0}^{t} \int_{L_{z}} v_{, 112}^{2} d x_{2} d \eta
$$$$
+\frac{a \lambda t^{2}}{2 \pi^{2}} \int_{0}^{t} \int_{L_{z}} u_{, 1 \eta}^{2} d x_{2} d \eta
$$

On combining the above computations with (16), we obtain

$$
|\Phi(z, t)| \leq k(t) \frac{\partial \Phi(z, t)}{\partial z}+\frac{\lambda \gamma}{2} \int_{0}^{t} \int_{L_{z}} v_{, \alpha \beta} v_{, \alpha \beta} d x_{2} d \eta,
$$

where

$$
k(t)=\max \left\{\frac{\widehat{k}_{1}(t)}{\mu}, \frac{\widehat{k}_{2}(t)}{\lambda \gamma}\right\}
$$

with

$\widehat{k}_{1}(t)$

$$
\begin{gathered}
=\gamma+\max \left\{\frac{\lambda^{2}+\mu h^{2}+2 a h^{2}+\gamma h^{2}+m^{2} h^{2}+\lambda \rho_{2} h^{2}}{\pi^{2}}\right. \\
\left.+\frac{\lambda m^{2}}{k}, \frac{t^{2}+2 a t^{2}+\lambda \rho_{2}+a \lambda t^{2}}{\pi^{2}}+\mu+\frac{m^{2}}{k}\right\}, \\
\widehat{k}_{2}(t)=\gamma+\frac{\gamma h^{2}}{\pi^{2}}+2 \lambda \gamma+\frac{\lambda h^{2} m^{2}}{\pi^{2} k}+\frac{\lambda \gamma h^{2}+a \lambda h^{2}}{\pi^{2}} .
\end{gathered}
$$


Using the method proposed in the paper [11], we define a new function:

$$
\begin{aligned}
M(z, t)= & \left.\frac{\rho_{1}}{4} \int_{L_{z}} u_{, \eta}^{2} d x_{2}\right|_{\eta=t}+\left.\frac{1}{4} \int_{L_{z}} u_{, \alpha} u_{, \alpha} d x_{2}\right|_{\eta=t} \\
& +\left.\frac{\rho_{2}}{4} \int_{L_{z}} v_{, \eta}^{2} d x_{2}\right|_{\eta=t}+\left.\frac{\gamma}{4} \int_{L_{z}} v_{, \alpha \beta} v_{, \alpha \beta} d x_{2}\right|_{\eta=t} \\
& +\left.\lambda \frac{m^{2}}{4 k} \int_{L_{z}} v_{, 1 \alpha} v_{, 1 \alpha} d x_{2}\right|_{\eta=t} .
\end{aligned}
$$

For $t>0$, let $\widetilde{t}$ be a value of time between 0 and $t$, at which function $M(z, t)$ gains its maximum value; that is,

$$
\begin{aligned}
M(z, \tilde{t})= & \left.\frac{\rho_{1}}{4} \int_{L_{z}} u_{, \eta}^{2} d x_{2}\right|_{\eta=\tilde{t}}+\left.\frac{1}{4} \int_{L_{z}} u_{, \alpha} u_{, \alpha} d x_{2}\right|_{\eta=\tilde{t}} \\
& +\left.\frac{\rho_{2}}{4} \int_{L_{z}} v_{, \eta}^{2} d x_{2}\right|_{\eta=\tilde{t}}+\left.\frac{\gamma}{4} \int_{L_{z}} v_{, \alpha \beta} v_{, \alpha \beta} d x_{2}\right|_{\eta=\tilde{t}} \\
& +\left.\lambda \frac{m^{2}}{4 k} \int_{L_{z}} v_{, 1 \alpha} v_{, 1 \alpha} d x_{2}\right|_{\eta=\tilde{t}} \\
= & \max _{0 \leq \tau \leq t}\left\{\left.\frac{\rho_{1}}{4} \int_{L_{z}} u_{, \eta}^{2} d x_{2}\right|_{\eta=\tau}+\left.\frac{1}{4} \int_{L_{z}} u_{, \alpha} u_{, \alpha} d x_{2}\right|_{\eta=\tau}\right. \\
& +\left.\frac{\rho_{2}}{4} \int_{L_{z}} v_{, \eta}^{2} d x_{2}\right|_{\eta=\tau} \\
& +\left.\frac{\gamma}{4} \int_{L_{z}} v_{, \alpha \beta} v_{, \alpha \beta} d x_{2}\right|_{\eta=\tau} \\
& \left.+\left.\lambda \frac{m^{2}}{4 k} \int_{L_{z}} v_{, 1 \alpha} v_{, 1 \alpha} d x_{2}\right|_{\eta=\tau}\right\}
\end{aligned}
$$

We now define

$$
t^{*}=\lim _{Z \rightarrow \infty}\left\{\sup _{0 \leq z \leq Z} \tilde{t}(z)\right\}
$$

From (22), we have

$$
\left|\Phi\left(z, t^{*}\right)\right| \leq k\left(t^{*}\right) \frac{\partial \Phi\left(z, t^{*}\right)}{\partial z}+\frac{\lambda \gamma}{2} \int_{0}^{t^{*}} \int_{L_{z}} v_{, \alpha \beta} v_{, \alpha \beta} d x_{2} d \eta
$$

From the definition of $t^{*}$, we have

$$
\begin{aligned}
& \frac{\lambda \gamma}{2} \int_{0}^{t^{*}} \int_{L_{z}} v_{, \alpha \beta} v_{, \alpha \beta} d x_{2} d \eta \\
& \quad \leq 2 \lambda \int_{0}^{t^{*}} M(z, t) d t \leq 2 \lambda t^{*} M\left(z, t^{*}\right) .
\end{aligned}
$$

On combining (20), (26), (28), and (29), we get

$$
\begin{aligned}
\left|\Phi\left(z, t^{*}\right)\right| & \leq k\left(t^{*}\right) \frac{\partial \Phi\left(z, t^{*}\right)}{\partial z}+\frac{\lambda \gamma}{2} \int_{0}^{t^{*}} \int_{L_{z}} v_{, \alpha \beta} v_{, \alpha \beta} d x_{2} d \eta \\
& \leq k\left(t^{*}\right) \frac{\partial \Phi\left(z, t^{*}\right)}{\partial z}+2 \lambda t^{*} M\left(z, t^{*}\right) \\
& \leq\left(k\left(t^{*}\right)+2 \lambda t^{*}\right) \frac{\partial \Phi\left(z, t^{*}\right)}{\partial z} \\
& \leq(k(t)+2 \lambda t) \frac{\partial \Phi\left(z, t^{*}\right)}{\partial z} .
\end{aligned}
$$

The following discussion will be divided into two cases.

Case 1. If there exists a point $\left(z_{1}, t^{*}\right)$ such that $\Phi\left(z_{1}, t^{*}\right)>0$, then for all $z>z_{1}, \Phi\left(z, t^{*}\right)>0$. An integration of (30) leads to

$$
\Phi\left(z, t^{*}\right) \geq \Phi\left(z_{1}, t^{*}\right) \exp \left(\frac{z-z_{1}}{k(t)+2 \lambda t}\right)
$$

Case 2. If there does not exist a point $\left(z_{1}, t^{*}\right)$ such that $\Phi\left(z_{1}, t^{*}\right)>0$, then for all $z>0, \Phi\left(z, t^{*}\right) \leq 0$. We have, from (30),

$$
-\Phi\left(z, t^{*}\right) \leq-\Phi\left(0, t^{*}\right) \exp [-(k(t)+2 \lambda t) z] .
$$

From (31), we can get $\lim _{z \rightarrow \infty} \Phi\left(z, t^{*}\right)=\infty$; that is to say, for $z$ large enough, we can get

$$
\Phi\left(z, t^{*}\right) \geq 2 \Phi\left(z_{0}, t^{*}\right)
$$

From (16), we get

$$
\Phi\left(z, t^{*}\right) \leq 2\left\{\left.\frac{\rho_{1}}{2} \int_{z_{0}}^{z} \int_{L_{\xi}} u_{, \eta}^{2} d A\right|_{\eta=t^{*}}\right.
$$

$$
\begin{aligned}
& +\left.\frac{1}{2} \int_{z_{0}}^{z} \int_{L_{\xi}} u_{, \alpha} u_{, \alpha} d A\right|_{\eta=t^{*}} \\
& +\mu \int_{0}^{t^{*}} \int_{z_{0}}^{z} \int_{L_{\xi}} u_{, \alpha \eta} u_{, \alpha \eta} d A d \eta
\end{aligned}
$$

$$
\begin{aligned}
& +\left.\frac{\rho_{2}}{2} \int_{z_{0}}^{z} \int_{L_{\xi}} v_{, \eta}^{2} d A\right|_{\eta=t^{*}} \\
& +\left.\frac{\gamma}{2} \int_{z_{0}}^{z} \int_{L_{\xi}} v_{, \alpha \beta} v_{, \alpha \beta} d A\right|_{\eta=t^{*}}
\end{aligned}
$$




$$
\begin{aligned}
& +\frac{m^{2}}{k} \int_{0}^{t^{*}} \int_{z_{0}}^{z} \int_{L_{\xi}} v_{, \alpha \eta} v_{, \alpha \eta} d A d \eta \\
& -\left.a \int_{z_{0}}^{z} \int_{L_{\xi}} u_{, \alpha} v_{, \alpha} d A\right|_{\eta=t^{*}} \\
& -\lambda \int_{0}^{t^{*}} \int_{z_{0}}^{z} \int_{L_{\xi}} \rho_{2} v_{, 1 \eta} v_{, 1 \eta} d A d \eta \\
& -\left.\lambda \int_{z_{0}}^{z} \int_{L_{\xi}} \rho_{2} v_{, 11} v_{, \eta} d A\right|_{\eta=t^{*}} \\
& +\lambda \gamma \int_{0}^{t^{*}} \int_{z_{0}}^{z} \int_{L_{\xi}} v_{, 1 \alpha \beta} v_{, 1 \alpha \beta} d A d \eta \\
& +\left.\lambda \frac{m^{2}}{2 k} \int_{z_{0}}^{z} \int_{L_{\xi}} v_{, 1 \alpha} v_{, 1 \alpha} d A\right|_{\eta=t^{*}} \\
& \left.+\lambda \int_{0}^{t^{*}} \int_{z_{0}}^{z} \int_{L_{\xi}} a v_{, 11 \alpha} u_{, \alpha} d A d \eta\right\}
\end{aligned}
$$

Following the same procedure as in deriving (20), we easily get

$$
\begin{aligned}
& \Phi\left(z, t^{*}\right) \leq 3\left\{\left.\frac{\rho_{1}}{2} \int_{z_{0}}^{z} \int_{L_{\xi}} u_{, \eta}^{2} d A\right|_{\eta=t^{*}}\right. \\
& +\left.\frac{1}{2} \int_{z_{0}}^{z} \int_{L_{\xi}} u_{, \alpha} u_{, \alpha} d A\right|_{\eta=t^{*}} \\
& +\mu \int_{0}^{t^{*}} \int_{z_{0}}^{z} \int_{L_{\xi}} u_{, \alpha \eta} u_{, \alpha \eta} d A d \eta \\
& +\left.\frac{\rho_{2}}{2} \int_{z_{0}}^{z} \int_{L_{\xi}} v_{, \eta}^{2} d A\right|_{\eta=t^{*}} \\
& +\left.\frac{\gamma}{2} \int_{z_{0}}^{z} \int_{L_{\xi}} v_{, \alpha \beta} v_{, \alpha \beta} d A\right|_{\eta=t^{*}} \\
& +\frac{m^{2}}{k} \int_{0}^{t^{*}} \int_{z_{0}}^{z} \int_{L_{\xi}} v_{, \alpha \eta} v_{, \alpha \eta} d A d \eta \\
& +\lambda \gamma \int_{0}^{t^{*}} \int_{z_{0}}^{z} \int_{L_{\xi}} v_{, 1 \alpha \beta} v_{, 1 \alpha \beta} d A d \eta \\
& \left.+\left.\lambda \frac{m^{2}}{2 k} \int_{z_{0}}^{z} \int_{L_{\xi}} v_{, 1 \alpha} v_{, 1 \alpha} d A\right|_{\eta=t^{*}}\right\} \\
& =F_{1}\left(z, t^{*}\right) \text {. }
\end{aligned}
$$

On combining (31) and (35), we get

$$
\exp \left(\frac{z_{1}-z}{k(t)+2 \lambda t}\right) F_{1}\left(z, t^{*}\right) \geq \Phi\left(z_{1}, t^{*}\right)
$$

Clearly, if $\lim _{z \rightarrow \infty} \Phi\left(z, t^{*}\right)=0$, we can easily get from (16) that

$$
\begin{aligned}
& -\Phi\left(z, t^{*}\right) \geq \frac{1}{2}\left\{\left.\frac{\rho_{1}}{2} \int_{z}^{\infty} \int_{L_{\xi}} u_{, \eta}^{2} d A\right|_{\eta=t^{*}}\right. \\
& +\left.\frac{1}{2} \int_{z}^{\infty} \int_{L_{\xi}} u_{, \alpha} u_{, \alpha} d A\right|_{\eta=t^{*}} \\
& +\mu \int_{0}^{t^{*}} \int_{z}^{\infty} \int_{L_{\xi}} u_{, \alpha \eta} u_{, \alpha \eta} d A d \eta \\
& +\left.\frac{\rho_{2}}{2} \int_{z}^{\infty} \int_{L_{\xi}} v_{, \eta}^{2} d A\right|_{\eta=t^{*}} \\
& +\left.\frac{\gamma}{2} \int_{z}^{\infty} \int_{L_{\xi}} v_{, \alpha \beta} v_{, \alpha \beta} d A\right|_{\eta=t^{*}} \\
& +\frac{m^{2}}{k} \int_{0}^{t^{*}} \int_{z}^{\infty} \int_{L_{\xi}} v_{, \alpha \eta} v_{, \alpha \eta} d A d \eta \\
& +\lambda \gamma \int_{0}^{t^{*}} \int_{z}^{\infty} \int_{L_{\xi}} v_{, 1 \alpha \beta} v_{, 1 \alpha \beta} d A d \eta \\
& \left.+\left.\lambda \frac{m^{2}}{2 k} \int_{z}^{\infty} \int_{L_{\xi}} v_{, 1 \alpha} v_{, 1 \alpha} d A\right|_{\eta=t^{*}}\right\} \\
& \geq \frac{1}{2}\left\{\left.\frac{\rho_{1}}{2} \int_{z}^{\infty} \int_{L_{\xi}} u_{, \eta}^{2} d A\right|_{\eta=t^{*}}\right. \\
& +\left.\frac{1}{2} \int_{z}^{\infty} \int_{L_{\xi}} u_{, \alpha} u_{, \alpha} d A\right|_{\eta=t^{*}} \\
& +\left.\frac{\rho_{2}}{2} \int_{z}^{\infty} \int_{L_{\xi}} v_{, \eta}^{2} d A\right|_{\eta=t^{*}} \\
& +\left.\frac{\gamma}{2} \int_{z}^{\infty} \int_{L_{\xi}} v_{, \alpha \beta} v_{, \alpha \beta} d A\right|_{\eta=t^{*}} \\
& \left.+\left.\lambda \frac{m^{2}}{2 k} \int_{z}^{\infty} \int_{L_{\xi}} v_{, 1 \alpha} v_{, 1 \alpha} d A\right|_{\eta=t^{*}}\right\} \\
& =F_{2}\left(z, t^{*}\right) \text {. }
\end{aligned}
$$

Summarizing all the results above, we conclude the following theorem.

Theorem 1. Let $(u, v)$ be classical solution of the initial boundary value problems (4)-(6); then, either

$$
\lim _{z \rightarrow \infty} \exp \left(\frac{z_{1}-z}{k(t)+2 \lambda t}\right) F_{1}\left(z, t^{*}\right) \geq \Phi\left(z_{1}, t^{*}\right)
$$


is satisfied or the "energy" function $F_{2}(z, t)$ satisfies the estimates

$$
\begin{aligned}
F_{2}(z, t) & \leq F_{2}\left(z, t^{*}\right) \leq-\Phi\left(0, t^{*}\right) \exp [-(k(t)+2 \lambda t) z] \\
& \leq-\Phi(0, t) \exp [-(k(t)+2 \lambda t) z],
\end{aligned}
$$

where $t^{*}$ is a value (see (27)) belonging to $(0, t]$.

\section{Spatial Decay Estimates}

In this part, we can give the spatial decay estimates based on a second-order differential inequality.

We add some conditions on the solutions

$$
\begin{gathered}
u, u_{, \eta}, u_{, \alpha} \longrightarrow 0 \quad \text { as } z \longrightarrow \infty, \\
v, v_{, \eta}, v_{, \alpha \eta}, v_{, \alpha \beta} \longrightarrow 0 \quad \text { as } z \longrightarrow \infty .
\end{gathered}
$$

From (4), we have

$$
\begin{aligned}
0= & \int_{0}^{t} \int_{z}^{\infty} \int_{L_{\xi}} \exp (-w \eta) u_{, \eta} \\
= & \frac{\rho_{1} w}{2} \int_{0}^{t} \int_{z}^{\infty} \int_{L_{\xi}} \exp (-w \eta) u_{, \eta}^{2} d A d \eta \\
& +\frac{\rho_{1}}{2} \int_{z}^{\infty} \int_{L_{\xi}} \exp (-w t) u_{,} u_{, t} d A \\
& +\int_{0}^{t} \int_{L_{z}}^{t} \exp (-w \eta) u_{, \alpha \alpha} u_{, 1} d x_{2} d \eta \\
& +\frac{w}{2} \int_{0}^{t} \int_{z}^{\infty} \int_{L_{\xi}} \exp (-w \eta) u_{, \alpha} u_{, \alpha} d A d \eta \\
& +\frac{1}{2} \int_{z}^{\infty} \int_{L_{\xi}} \exp (-w t) u_{, \alpha} u_{, \alpha} d A \\
& +\mu \int_{0}^{t} \int_{z}^{\infty} \int_{L_{\xi}} \exp (-w \eta) u_{, \alpha \eta} u_{, \alpha \eta} d A d \eta \\
& +\mu \int_{0}^{t} \int_{L_{z}} \exp (-w \eta) u_{, \eta} u_{, 1 \eta} d x_{2} d \eta \\
& +a \int_{0}^{t} \int_{z}^{\infty} \int_{L_{\xi}} \exp (-w \eta) u_{, \eta} v_{, \alpha \alpha} d A d \eta .
\end{aligned}
$$

From (5), we also have

$$
\begin{gathered}
0=\int_{0}^{t} \int_{z}^{\infty} \int_{L_{\xi}} \exp (-w \eta) v_{, \eta}\left(\rho_{2} v_{, \eta \eta}+\gamma v_{, \alpha \alpha \beta \beta}\right. \\
\left.+a u_{, \alpha \alpha}-\frac{m^{2}}{k} v_{, \alpha \alpha \eta}\right) d A d \eta \\
=\frac{\rho_{2} w}{2} \int_{0}^{t} \int_{z}^{\infty} \int_{L_{\xi}} \exp (-w \eta) v_{, \eta}^{2} d A d \eta
\end{gathered}
$$

$$
\begin{aligned}
& +\frac{\rho_{2}}{2} \int_{z}^{\infty} \int_{L_{\xi}} \exp (-w t) v_{, t} v_{, t} d A \\
& +\frac{w \gamma}{2} \int_{0}^{t} \int_{z}^{\infty} \int_{L_{\xi}} \exp (-w \eta) v_{, \alpha \beta} v_{, \alpha \beta} d A d \eta \\
& +\frac{\gamma}{2} \int_{z}^{\infty} \int_{L_{\xi}} \exp (-w t) v_{, \alpha \beta} v_{, \alpha \beta} d A \\
& +\gamma \int_{0}^{t} \int_{L_{z}} \exp (-w \eta) v_{, \alpha \eta} v_{, \alpha 1} d x_{2} d \eta \\
& -\gamma \int_{0}^{t} \int_{L_{z}} \exp (-w \eta) v_{, \eta} v_{, 1 \beta \beta} d x_{2} d \eta \\
& +a \int_{0}^{t} \int_{z}^{\infty} \int_{L_{\xi}} \exp (-w \eta) v_{, \eta} u_{, \alpha \alpha} d A d \eta \\
& +\frac{m^{2}}{k} \int_{0}^{t} \int_{z}^{\infty} \int_{L_{\xi}} \exp (-w \eta) v_{, \alpha \eta} v_{, \alpha \eta} d A d \eta \\
& +\frac{m^{2}}{k} \int_{0}^{t} \int_{L_{z}} \exp (-w \eta) v_{, \eta} v_{, 1 \eta} d A d \eta .
\end{aligned}
$$

We now tackle the item

$$
\begin{aligned}
a \int_{0}^{t} \int_{z}^{\infty} \int_{L_{\xi}} \exp (-w \eta) v_{, \eta} u_{, \alpha \alpha} d A d \eta \\
=-a \int_{0}^{t} \int_{z}^{\infty} \int_{L_{\xi}} \exp (-w \eta) v_{, \alpha \eta} u_{, \alpha} d A d \eta \\
\quad-a \int_{0}^{t} \int_{L_{z}} \exp (-w \eta) v_{, \eta} u_{, 1} d x_{2} d \eta \\
=a \int_{0}^{t} \int_{z}^{\infty} \int_{L_{\xi}}^{\infty} \exp (-w \eta) v_{, \alpha \alpha \eta} u d A d \eta \\
\quad-a \int_{0}^{t} \int_{L_{z}} \exp (-w \eta) v_{, \eta} u_{, 1} d x_{2} d \eta \\
\quad+a \int_{0}^{t} \int_{L_{z}} \exp (-w \eta) v_{, 1 \eta} u d x_{2} d \eta \\
=-a \int_{0}^{t} \int_{z}^{\infty} \int_{L_{\xi}} \exp (-w \eta) v_{, \alpha \alpha} u_{, \eta} d A d \eta \\
\quad+a w \int_{0}^{t} \int_{z}^{\infty} \int_{L_{\xi}}^{\infty} \exp (-w \eta) v_{, \alpha \alpha} u d A d \eta \\
\quad+a \int_{z}^{\infty} \int_{L_{\xi}} \exp (-w t) v_{, \alpha \alpha} u d A
\end{aligned}
$$




$$
\begin{aligned}
& -a \int_{0}^{t} \int_{L_{z}} \exp (-w \eta) v_{, \eta} u_{, 1} d x_{2} d \eta \\
& +a \int_{0}^{t} \int_{L_{z}} \exp (-w \eta) v_{, 1 \eta} u d x_{2} d \eta
\end{aligned}
$$

If we define a new function

$$
\begin{aligned}
& E(z, t)=\frac{\rho_{1} w}{2} \int_{0}^{t} \int_{z}^{\infty} \int_{L_{\xi}} \exp (-w \eta) u_{, \eta}^{2} d A d \eta \\
& +\frac{\rho_{1}}{2} \int_{z}^{\infty} \int_{L_{\xi}} \exp (-w t) u_{, t} u_{, t} d A \\
& +\frac{w}{2} \int_{0}^{t} \int_{z}^{\infty} \int_{L_{\xi}} \exp (-w \eta) u_{, \alpha} u_{, \alpha} d A d \eta \\
& +\frac{1}{2} \int_{z}^{\infty} \int_{L_{\xi}} \exp (-w t) u_{, \alpha} u_{, \alpha} d A \\
& +\mu \int_{0}^{t} \int_{z}^{\infty} \int_{L_{\xi}} \exp (-w \eta) u_{, \alpha \eta} u_{, \alpha \eta} d A d \eta \\
& +\frac{\rho_{2} w}{2} \int_{0}^{t} \int_{z}^{\infty} \int_{L_{\xi}} \exp (-w \eta) v_{, \eta}^{2} d A d \eta \\
& +\frac{\rho_{2}}{2} \int_{z}^{\infty} \int_{L_{\xi}} \exp (-w t) v_{, t} v_{, t} d A \\
& +\frac{w \gamma}{2} \int_{0}^{t} \int_{z}^{\infty} \int_{L_{\xi}} \exp (-w \eta) v_{, \alpha \beta} v_{, \alpha \beta} d A d \eta \\
& +\frac{\gamma}{2} \int_{z}^{\infty} \int_{L_{\xi}} \exp (-w t) v_{, \alpha \beta} v_{, \alpha \beta} d A \\
& +\frac{m^{2}}{k} \int_{0}^{t} \int_{z}^{\infty} \int_{L_{\xi}} \exp (-w \eta) v_{, \alpha \eta} v_{, \alpha \eta} d A d \eta \\
& +a w \int_{0}^{t} \int_{z}^{\infty} \int_{L_{\xi}} \exp (-w \eta) v_{, \alpha \alpha} u d A d \eta \\
& +a \int_{z}^{\infty} \int_{L_{\xi}} \exp (-w t) v_{, \alpha \alpha} u d A,
\end{aligned}
$$

on combining (44), (42), and (43), we can easily get

$$
\begin{aligned}
E(z, t)= & -\int_{0}^{t} \int_{L_{z}} \exp (-w \eta) u_{, \eta} u_{, 1} d x_{2} d \eta \\
& -\mu \int_{0}^{t} \int_{L_{z}} \exp (-w \eta) u_{, \eta} u_{, 1 \eta} d x_{2} d \eta \\
& -\gamma \int_{0}^{t} \int_{L_{z}} \exp (-w \eta) v_{, \alpha \eta} v_{, 1 \alpha} d x_{2} d \eta \\
& +\gamma \int_{0}^{t} \int_{L_{z}} \exp (-w \eta) v_{, \eta} v_{, 1 \beta \beta} d x_{2} d \eta \\
& -\frac{m^{2}}{k} \int_{0}^{t} \int_{L_{z}} \exp (-w \eta) v_{, \eta} v_{, 1 \eta} d x_{2} d \eta
\end{aligned}
$$

$$
\begin{aligned}
& +a \int_{0}^{t} \int_{L_{z}} \exp (-w \eta) v_{, \eta} u_{, 1} d x_{2} d \eta \\
& -a \int_{0}^{t} \int_{L_{z}} \exp (-w \eta) v_{, 1 \eta} u d x_{2} d \eta
\end{aligned}
$$

Using the Cauchy inequality, we obtain

$$
\begin{gathered}
\left|a w \int_{0}^{t} \int_{z}^{\infty} \int_{L_{\xi}} \exp (-w \eta) v_{, \alpha \alpha} u d A d \eta\right| \\
\leq \frac{w \gamma}{8} \int_{0}^{t} \int_{z}^{\infty} \int_{L_{\xi}} \exp (-w \eta) v_{, \alpha \beta} v_{, \alpha \beta} d A d \eta \\
\quad+\frac{2 a^{2} w h^{2}}{\gamma \pi^{2}} \int_{0}^{t} \int_{z}^{\infty} \int_{L_{\xi}} \exp (-w \eta) u_{, \alpha} u_{, \alpha} d A d \eta \\
\left|a \int_{z}^{\infty} \int_{L_{\xi}} \exp (-w t) v_{, \alpha \alpha} u d A\right| \\
\leq \frac{\gamma}{8} \int_{z}^{\infty} \int_{L_{\xi}} \exp (-w t) v_{, \alpha \beta} v_{, \alpha \beta} d A \\
+\frac{2 a^{2} h^{2}}{\gamma \pi^{2}} \int_{z}^{\infty} \int_{L_{\xi}} \exp (-w t) u_{, \alpha} u_{, \alpha} d A .
\end{gathered}
$$

If we choose suitable $a$ such that

$$
\frac{a^{2} h^{2}}{\pi^{2} \gamma} \leq \frac{1}{8}
$$

on combining (44)-(46), we have

$$
\begin{aligned}
E(z, t) \geq & \frac{\rho_{1} w}{4} \int_{0}^{t} \int_{z}^{\infty} \int_{L_{\xi}} \exp (-w \eta) u_{, \eta}^{2} d A d \eta \\
& +\frac{\rho_{1}}{4} \int_{z}^{\infty} \int_{L_{\xi}} \exp (-w t) u_{,} u_{,} d A \\
& +\frac{w}{4} \int_{0}^{t} \int_{z}^{\infty} \int_{L_{\xi}} \exp (-w \eta) u_{, \alpha} u_{, \alpha} d A d \eta \\
& +\frac{1}{4} \int_{z}^{\infty} \int_{L_{\xi}} \exp (-w t) u_{, \alpha} u_{, \alpha} d A \\
& +\frac{\mu}{2} \int_{0}^{t} \int_{z}^{\infty} \int_{L_{\xi}} \exp (-w \eta) u_{, \alpha \eta} u_{, \alpha \eta} d A d \eta \\
& +\frac{\rho_{2} w}{4} \int_{0}^{t} \int_{z}^{\infty} \int_{L_{\xi}} \exp (-w \eta) v_{, \eta}^{2} d A d \eta \\
& +\frac{\rho_{2}}{4} \int_{z}^{\infty} \int_{L_{\xi}} \exp (-w t) v_{, t} v_{, t} d A \\
& +\frac{w \gamma}{4} \int_{0}^{t} \int_{z}^{\infty} \int_{L_{\xi}} \exp (-w \eta) v_{, \alpha \beta} v_{, \alpha \beta} d A d \eta
\end{aligned}
$$




$$
\begin{aligned}
& +\frac{\gamma}{4} \int_{z}^{\infty} \int_{L_{\xi}} \exp (-w t) v_{, \alpha \beta} v_{, \alpha \beta} d A \\
& +\frac{m^{2}}{2 k} \int_{0}^{t} \int_{z}^{\infty} \int_{L_{\xi}} \exp (-w \eta) v_{, \alpha \eta} v_{, \alpha \eta} d A d \eta
\end{aligned}
$$

We define

$$
\begin{aligned}
& F(z, t)=\int_{z}^{\infty} E(\xi, t) d \xi \\
& =-\int_{0}^{t} \int_{z}^{\infty} \int_{L_{\xi}} \exp (-w \eta) u_{, \eta} u_{, 1} d A d \eta \\
& -\mu \int_{0}^{t} \int_{z}^{\infty} \int_{L_{\xi}} \exp (-w \eta) u_{, \eta} u_{, 1 \eta} d A d \eta \\
& -\gamma \int_{0}^{t} \int_{z}^{\infty} \int_{L_{\xi}} \exp (-w \eta) v_{, \alpha \eta} v_{, 1 \alpha} d A d \eta \\
& +\gamma \int_{0}^{t} \int_{z}^{\infty} \int_{L_{\xi}} \exp (-w \eta) v_{, \eta} v_{, 1 \beta \beta} d A d \eta \\
& -\frac{m^{2}}{k} \int_{0}^{t} \int_{z}^{\infty} \int_{L_{\xi}} \exp (-w \eta) v_{, \eta} v_{, 1 \eta} d A d \eta \\
& +a \int_{0}^{t} \int_{z}^{\infty} \int_{L_{\xi}} \exp (-w \eta) v_{, \eta} u_{, 1} d A d \eta \\
& -a \int_{0}^{t} \int_{z}^{\infty} \int_{L_{\xi}} \exp (-w \eta) v_{, 1 \eta} u d A d \eta .
\end{aligned}
$$

On combining (44) and (49), we also have

$$
\begin{aligned}
& F(z, t)=\frac{\rho_{1} w}{2} \int_{0}^{t} \int_{z}^{\infty} \int_{L_{\xi}}(\xi-z) \exp (-w \eta) u_{, \eta}^{2} d A d \eta \\
& +\frac{\rho_{1}}{2} \int_{z}^{\infty} \int_{L_{\xi}}(\xi-z) \exp (-w t) u_{, t} u_{, t} d A \\
& +\frac{w}{2} \int_{0}^{t} \int_{z}^{\infty} \int_{L_{\xi}}(\xi-z) \exp (-w \eta) u_{, \alpha} u_{, \alpha} d A d \eta \\
& +\frac{1}{2} \int_{z}^{\infty} \int_{L_{\xi}}(\xi-z) \exp (-w t) u_{, \alpha} u_{, \alpha} d A \\
& +\mu \int_{0}^{t} \int_{z}^{\infty} \int_{L_{\xi}}(\xi-z) \exp (-w \eta) u_{, \alpha \eta} u_{, \alpha \eta} d A d \eta \\
& +\frac{\rho_{2} w}{2} \int_{0}^{t} \int_{z}^{\infty} \int_{L_{\xi}}(\xi-z) \exp (-w \eta) v_{, \eta}^{2} d A d \eta
\end{aligned}
$$

$$
\begin{aligned}
& +\frac{\rho_{2}}{2} \int_{z}^{\infty} \int_{L_{\xi}}(\xi-z) \exp (-w t) v_{, t} v_{, t} d A \\
& +\frac{w \gamma}{2} \int_{0}^{t} \int_{z}^{\infty} \int_{L_{\xi}}(\xi-z) \exp (-w \eta) v_{, \alpha \beta} v_{, \alpha \beta} d A d \eta \\
& +\frac{\gamma}{2} \int_{z}^{\infty} \int_{L_{\xi}}(\xi-z) \exp (-w t) v_{, \alpha \beta} v_{, \alpha \beta} d A \\
& +\frac{m^{2}}{k} \int_{0}^{t} \int_{z}^{\infty} \int_{L_{\xi}}(\xi-z) \exp (-w \eta) v_{, \alpha \eta} v_{, \alpha \eta} d A d \eta \\
& +a w \int_{0}^{t} \int_{z}^{\infty} \int_{L_{\xi}}(\xi-z) \exp (-w \eta) v_{, \alpha \alpha} u d A d \eta \\
& +a \int_{z}^{\infty} \int_{L_{\xi}}(\xi-z) \exp (-w t) v_{, \alpha \alpha} u d A .
\end{aligned}
$$

Following the Schwarz inequality, we can easily get

$$
\begin{aligned}
& F(z, t) \geq \frac{\rho_{1} w}{4} \int_{0}^{t} \int_{z}^{\infty} \int_{L_{\xi}}(\xi-z) \exp (-w \eta) u_{, \eta}^{2} d A d \eta \\
& +\frac{\rho_{1}}{4} \int_{z}^{\infty} \int_{L_{\xi}}(\xi-z) \exp (-w t) u_{, t} u_{, t} d A \\
& +\frac{w}{4} \int_{0}^{t} \int_{z}^{\infty} \int_{L_{\xi}}(\xi-z) \exp (-w \eta) u_{, \alpha} u_{, \alpha} d A d \eta \\
& +\frac{1}{4} \int_{z}^{\infty} \int_{L_{\xi}}(\xi-z) \exp (-w t) u_{, \alpha} u_{, \alpha} d A \\
& +\frac{\mu}{2} \int_{0}^{t} \int_{z}^{\infty} \int_{L_{\xi}}(\xi-z) \exp (-w \eta) u_{, \alpha \eta} u_{, \alpha \eta} d A d \eta \\
& +\frac{\rho_{2} w}{4} \int_{0}^{t} \int_{z}^{\infty} \int_{L_{\xi}}(\xi-z) \exp (-w \eta) v_{, \eta}^{2} d A d \eta \\
& +\frac{\rho_{2}}{4} \int_{z}^{\infty} \int_{L_{\xi}}(\xi-z) \exp (-w t) v_{, t} v_{, t} d A \\
& +\frac{w \gamma}{4} \int_{0}^{t} \int_{z}^{\infty} \int_{L_{\xi}}(\xi-z) \exp (-w \eta) v_{, \alpha \beta} v_{, \alpha \beta} d A d \eta \\
& +\frac{\gamma}{4} \int_{z}^{\infty} \int_{L_{\xi}}(\xi-z) \exp (-w t) v_{, \alpha \beta} v_{, \alpha \beta} d A \\
& +\frac{m^{2}}{2 k} \int_{0}^{t} \int_{z}^{\infty} \int_{L_{\xi}}(\xi-z) \exp (-w \eta) v_{, \alpha \eta} v_{, \alpha \eta} d A d \eta
\end{aligned}
$$


and we also get

$$
\begin{aligned}
& \frac{\partial F(z, t)}{\partial z}=-E(z, t), \\
& \frac{\partial^{2} F(z, t)}{\partial z^{2}} \geq \frac{\rho_{1} w}{4} \int_{0}^{t} \int_{L_{z}} \exp (-w \eta) u_{, \eta}^{2} d A d \eta \\
& +\frac{\rho_{1}}{4} \int_{L_{z}} \exp (-w t) u_{, t} u_{, t} d x_{2} \\
& +\frac{w}{4} \int_{0}^{t} \int_{L_{z}} \exp (-w \eta) u_{, \alpha} u_{, \alpha} d x_{2} d \eta \\
& +\frac{1}{4} \int_{L_{z}} \exp (-w t) u_{, \alpha} u_{, \alpha} d x_{2} \\
& +\frac{\mu}{2} \int_{0}^{t} \int_{L_{z}} \exp (-w \eta) u_{, \alpha \eta} u_{, \alpha \eta} d x_{2} d \eta \\
& +\frac{\rho_{2} w}{4} \int_{0}^{t} \int_{L_{z}} \exp (-w \eta) v_{, \eta}^{2} d x_{2} d \eta \\
& +\frac{\rho_{2}}{4} \int_{L_{z}} \exp (-w t) v_{, t} v_{, t} d x_{2} \\
& +\frac{w \gamma}{4} \int_{0}^{t} \int_{L_{z}} \exp (-w \eta) v_{, \alpha \beta} v_{, \alpha \beta} d x_{2} d \eta \\
& +\frac{\gamma}{4} \int_{L_{z}} \exp (-w t) v_{, \alpha \beta} v_{, \alpha \beta} d x_{2} \\
& +\frac{m^{2}}{2 k} \int_{0}^{t} \int_{L_{z}} \exp (-w \eta) v_{, \alpha \eta} v_{, \alpha \eta} d x_{2} d \eta \text {. }
\end{aligned}
$$

Our goal in this part is to derive a differential inequality for the energy $F(z, t)$ of the form

$$
\frac{\partial^{2} F}{\partial z^{2}}-\gamma_{1} \frac{\partial F}{\partial z}-\gamma_{2} F \geq 0, \quad \gamma_{1}, \gamma_{2}>0
$$

From the definition of $F(z, t)$ in (49), using the Schwarz inequality and (52), we can get

$$
\begin{aligned}
& -\int_{0}^{t} \int_{z}^{\infty} \int_{L_{\xi}} \exp (-w \eta) u_{, \eta} u_{, 1} d A d \eta \\
& -\mu \int_{0}^{t} \int_{z}^{\infty} \int_{L_{\xi}} \exp (-w \eta) u_{, \eta} u_{, 1 \eta} d A d \eta \\
& -\gamma \int_{0}^{t} \int_{z}^{\infty} \int_{L_{\xi}} \exp (-w \eta) v_{, \alpha \eta} v_{, 1 \alpha} d A d \eta \\
& \quad-\frac{m^{2}}{k} \int_{0}^{t} \int_{z}^{\infty} \int_{L_{\xi}} \exp (-w \eta) v_{, \eta} v_{, 1 \eta} d A d \eta
\end{aligned}
$$

$$
\begin{aligned}
& +a \int_{0}^{t} \int_{z}^{\infty} \int_{L_{\xi}} \exp (-w \eta) v_{, \eta} u_{, 1} d A d \eta \\
& -a \int_{0}^{t} \int_{z}^{\infty} \int_{L_{\xi}} \exp (-w \eta) v_{, 1 \eta} u d A d \eta \\
& \leq\left(-k_{1}\right) \frac{\partial F}{\partial z}
\end{aligned}
$$

where $k_{1}$ is a positive constant.

In order to give a bound for $F$, we only need to give a bound for $\gamma \int_{0}^{t} \int_{z}^{\infty} \int_{L_{\xi}} \exp (-w \eta) v_{, \eta} v_{, 1 \beta \beta} d A d \eta$. We know that

$$
\begin{aligned}
& \gamma \int_{0}^{t} \int_{z}^{\infty} \int_{L_{\xi}} \exp (-w \eta) v_{, \eta} v_{, 1 \beta \beta} d A d \eta \\
& =-\gamma \int_{0}^{t} \int_{z}^{\infty} \int_{L_{\xi}} \exp (-w \eta) v_{, \beta \eta} v_{, 1 \beta} d A d \eta \\
& \quad-\gamma \int_{0}^{t} \int_{L_{z}} \exp (-w \eta) v_{, \eta} v_{, 11} d x_{2} d \eta
\end{aligned}
$$

Using the Schwarz inequality (52) and (53), we obtain

$$
\left|\gamma \int_{0}^{t} \int_{z}^{\infty} \int_{L_{\xi}} \exp (-w \eta) v_{, \eta} v_{, 1 \beta \beta} d A d \eta\right| \leq\left(-k_{2}\right) \frac{\partial F}{\partial z}+k_{3} \frac{\partial^{2} F}{\partial z^{2}}
$$

with $k_{2}$ and $k_{3}$ being all positive constants.

A combination of (55) and (57) leads to the result

$$
F \leq\left(-k_{1}-k_{2}\right) \frac{\partial F}{\partial z}+k_{3} \frac{\partial^{2} F}{\partial z^{2}}
$$

If we set $\gamma_{1}=\left(k_{1}+k_{2}\right) / k_{3}$ and $\gamma_{2}=1 / k_{3}$, we can get the result (54).

Inequality (54) may be rewritten as

$$
\left(\frac{\partial}{\partial z}-a\right)\left(\frac{\partial F(z, t)}{\partial z}+b F(z, t)\right) \geq 0
$$

where $a=\left(\sqrt{\gamma_{1}^{2}+4 \gamma_{2}}+\gamma_{1}\right) / 2, b=\left(\sqrt{\gamma_{1}^{2}+4 \gamma_{2}}-\gamma_{1}\right) / 2$.

From (59), we have

$$
\frac{\partial}{\partial z}\left[e^{-a z}\left(\frac{\partial F(z, t)}{\partial z}+b F(z, t)\right)\right] \geq 0
$$

Integrating (60) with respect to $z$ from $z$ to $\infty$, we obtain

$$
\frac{\partial F(z, t)}{\partial z}+b F(z, t) \leq 0 .
$$

We can easily get

$$
F(z, t) \leq F(0, t) e^{-b z}
$$

Theorem 2. Let $(u, v)$ be classical solution of the initial boundary value problems (4)-(6); the solutions satisfy conditions (40). One can get the decay estimates

$$
F(z, t) \leq F(0, t) e^{-b z}
$$




\section{Conflict of Interests}

The authors declare that there is no conflict of interests regarding the publication of this paper.

\section{Acknowledgments}

The work was supported by the national natural Science Foundation of China (no. 11201087), Foundation for Distinguished Young Talents in Higher Education of Guangdong, China (no. 2012LYM_0098), Foundation for Technology Innovation in Higher Education of Guangdong, China (no. 2013KJCX0136), the Excellent Young Teachers Training Program for Colleges and Universities of Guangdong Province, China (no. Yq2013121), the Natural Science Foundation of Hunan Province (no. 14JJ4044), and the Natural Science Foundation of Hunan Province (no. 13JJ3085).

\section{References}

[1] C. O. Horgan, "Recent developments concerning Saint-Venant's principle: an update," Applied Mechanics Reviews, vol. 42, no. 11, pp. 295-303, 1989.

[2] C. O. Horgan, "Recent developments concerning Saint-Venant's principle: a second update," Applied Mechanics Reviews, vol. 49, no. 10, pp. S101-S111, 1996.

[3] C. O. Horgan and J. K. Knowles, "Recent development concerning Saint-Venant's principle," in Advance in Applied Mechanics, J. W. Hutchinson and T. Y. Wu, Eds., vol. 23, pp. 179-269, Academic Press, New York, NY, USA, 1983.

[4] C. D’Apice, "Convexity considerations and spatial behavior for the harmonic vibrations in thermoelastic plates," Journal of Mathematical Analysis and Applications, vol. 312, no. 1, pp. 4460, 2005.

[5] C. D’Apice, "On a generalized biharmonic equation in plane polars with applications to functionally graded materials," The Australian Journal of Mathematical Analysis and Applications, vol. 3, no. 2, pp. 1-15, 2006.

[6] S. Chiriţă and C. D’Apice, "On spatial growth or decay of solutions to a non-simple heat conduction problem in a semiinfinite strip," Analele Stiintifice ale Universitatii "Alexandru Ioan Cuza" din Iasi, Matematica, vol. 48, no. 1, pp. 75-100, 2002.

[7] S. Chiriţă, M. Ciarletta, and M. Fabrizio, "Some spatial decay estimates in time-dependent Stokes slow flows," Applicable Analysis, vol. 77, no. 3-4, pp. 211-231, 2001.

[8] M. Fabrizio and S. Chiriţă, "Some qualitative results on the dynamic viscoelasticity of the Reissner-Mindlin plate model," The Quarterly Journal of Mechanics and Applied Mathematics, vol. 57, no. 1, pp. 59-78, 2004.

[9] W. S. Edelstein, "A spatial decay estimate for the heat equation," Journal of Applied Mathematics and Physics (ZAMP), vol. 20, pp. 900-905, 1969.

[10] C. H. Lin, "Spatial decay estimates and energy bounds for the stokes flow equation," Stability and Applied Analysis of Continuous Media, vol. 2, pp. 249-264, 1992.

[11] C. H. Lin and L. E. Payne, "A Phragmén-Lindelöf alternative for a class of quasilinear second order parabolic problems," Differential and Integral Equations, vol. 8, no. 3, pp. 539-551, 1995.
[12] R. J. Knops and L. E. Payne, "A Saint-Venant principle for nonlinear elasticity" Archive for Rational Mechanics and Analysis, vol. 81, no. 1, pp. 1-12, 1983.

[13] A. Borrelli and M. C. Patria, "Energy bounds in dynamical problems for a semi-infinite magnetoelastic beam," Journal of Applied Mathematics and Physics (ZAMP), vol. 47, no. 6, pp. 880-893, 1996.

[14] J. I. Diaz and R. Quintanilla, "Spatial and continuous dependence estimates in linear viscoelasticity," Journal of Mathematical Analysis and Applications, vol. 273, no. 1, pp. 1-16, 2002.

[15] R. Quintanilla, "A spatial decay estimate for the hyperbolic heat equation," SIAM Journal on Mathematical Analysis, vol. 27, no. 1, pp. 78-91, 1996.

[16] R. Quintanilla, "Phragmen-Lindelof alternative in nonlinear viscoelasticity," Nonlinear Analysis. Theory, Methods \& Applications A, vol. 34, no. 1, pp. 7-16, 1998.

[17] J. K. Knowles, "On Saint-Venant's principle in the twodimensional linear theory of elasticity," Archive for Rational Mechanics and Analysis, vol. 21, pp. 1-22, 1966.

[18] J. K. Knowles, "An energy estimate for the biharmonic equation and its application to Saint-Venant's principle in plane elastostatics," Indian Journal of Pure and Applied Mathematics, vol. 14, no. 7, pp. 791-805, 1983.

[19] J. N. Flavin, “On Knowles' version of Saint-Venant's principle in two-dimensional elastostatics," Archive for Rational Mechanics and Analysis, vol. 53, pp. 366-375, 1973/74.

[20] J. N. Flavin and R. J. Knops, "Some convexity considerations for a two-dimensional traction problem," Journal of Applied Mathematics and Physics (ZAMP), vol. 39, no. 2, pp. 166-176, 1988.

[21] C. O. Horgan, "Decay estimates for the biharmonic equation with applications to Saint-Venant's principles in plane elasticity and Stokes flows," Quarterly of Applied Mathematics, vol. 47, no. 1, pp. 147-157, 1989.

[22] L. E. Payne and P. W. Schaefer, "Some Phragmén-Lindelöf type results for the biharmonic equation," Journal of Applied Mathematics and Physics (ZAMP), vol. 45, no. 3, pp. 414-432, 1994.

[23] V. Varlamov, "Existence and uniqueness of a solution to the Cauchy problem for the damped Boussinesq equation," Mathematical Methods in the Applied Sciences, vol. 19, no. 8, pp. 639649, 1996.

[24] R. J. Knops and C. Lupoli, "End effects for plane Stokes flow along a semi-infinite strip," Journal of Applied Mathematics and Physics (ZAMP), vol. 48, no. 6, pp. 905-920, 1997.

[25] J. C. Song, "Improved decay estimates in time-dependent Stokes flow," Journal of Mathematical Analysis and Applications, vol. 288, no. 2, pp. 505-517, 2003.

[26] J. C. Song, "Improved spatial decay bounds in the plane Stokes flow," Applied Mathematics and Mechanics. English Edition, vol. 30, no. 7, pp. 833-838, 2009.

[27] M. L. Santos and J. E. Muñoz Rivera, "Analytic property of a coupled system of wave-plate type with thermal effect," Differential and Integral Equations, vol. 24, no. 9-10, pp. 965972, 2011.

[28] A. E. H. Love, Mathematical Theory of Elasticity, Dover, New York, NY, USA, 4th edition, 1942. 


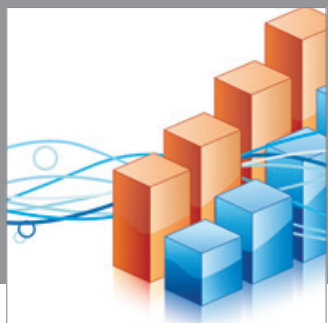

Advances in

Operations Research

mansans

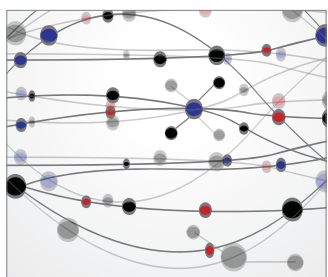

The Scientific World Journal
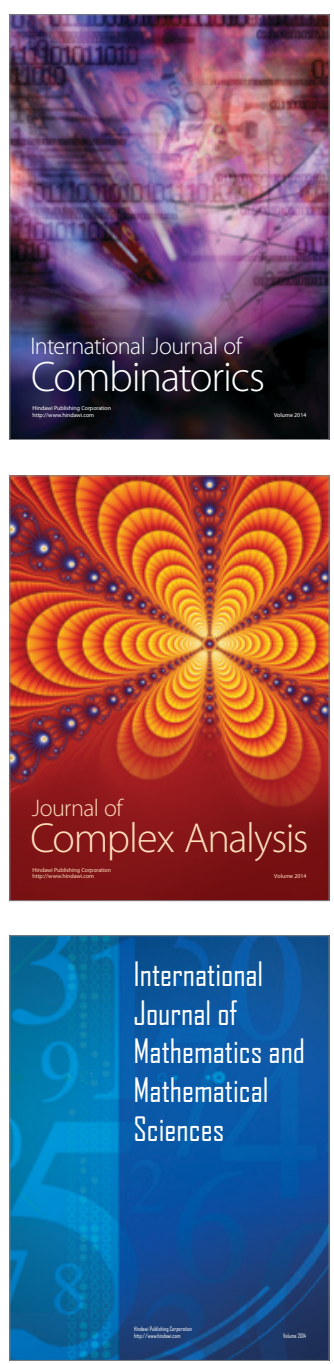
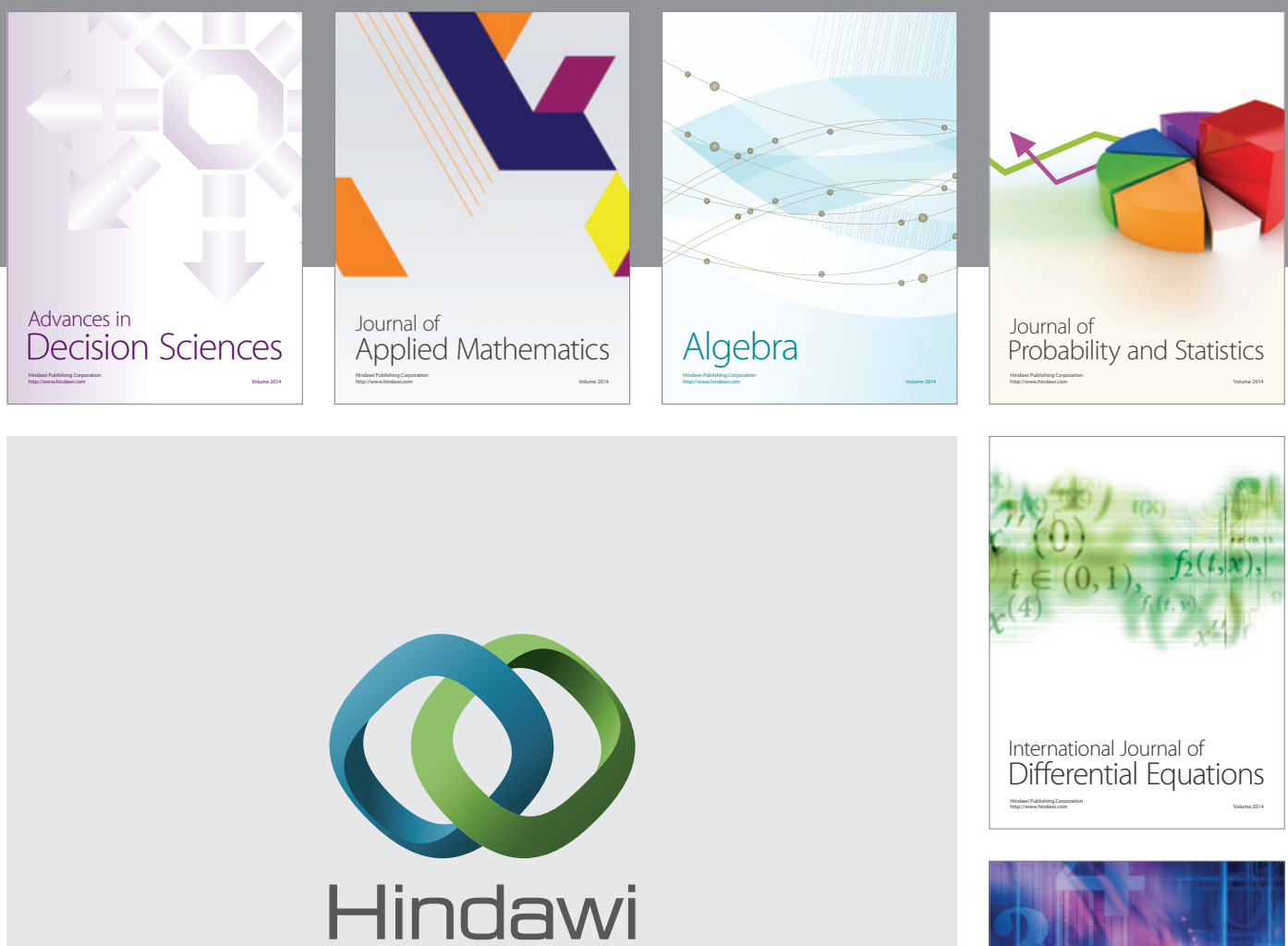

Submit your manuscripts at http://www.hindawi.com
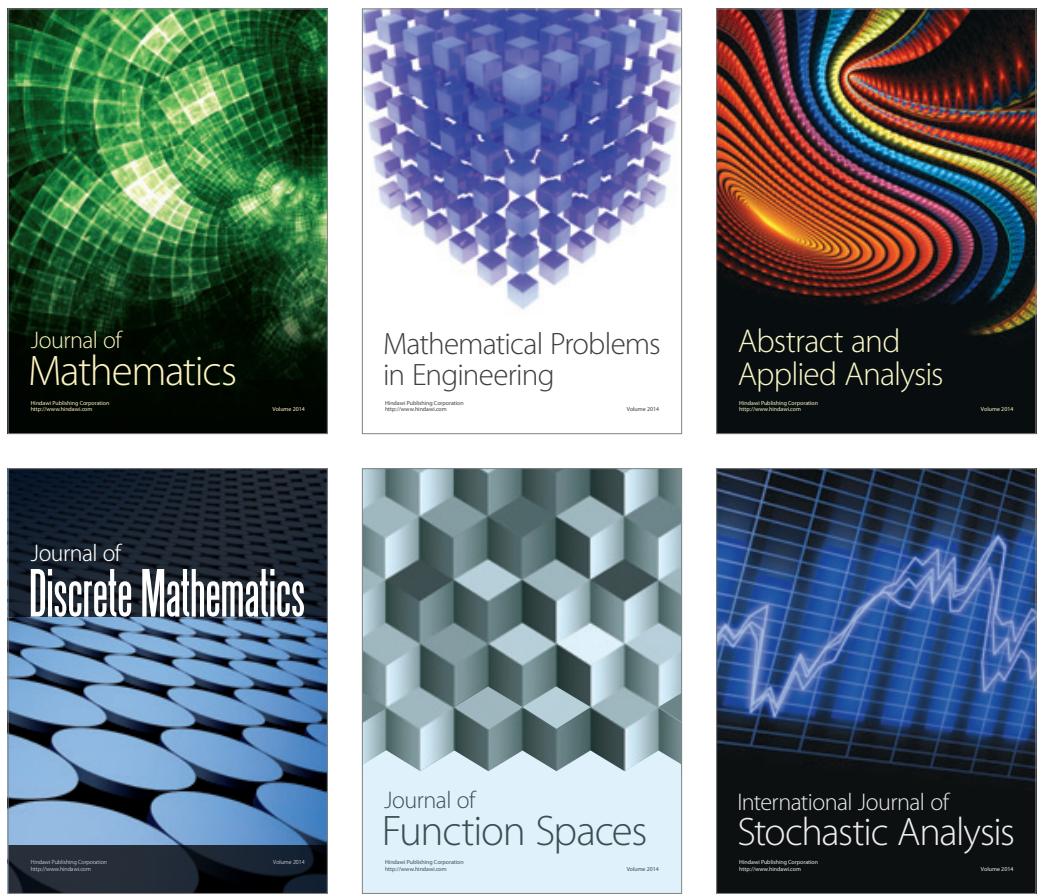

Journal of

Function Spaces

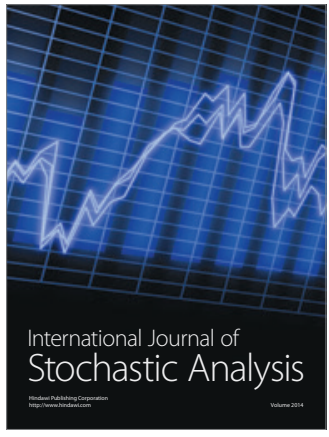

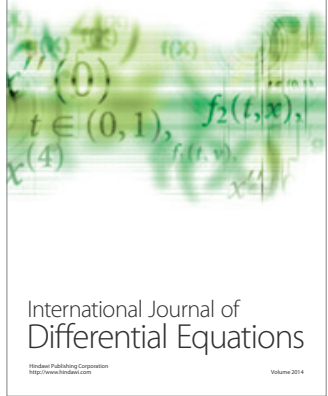
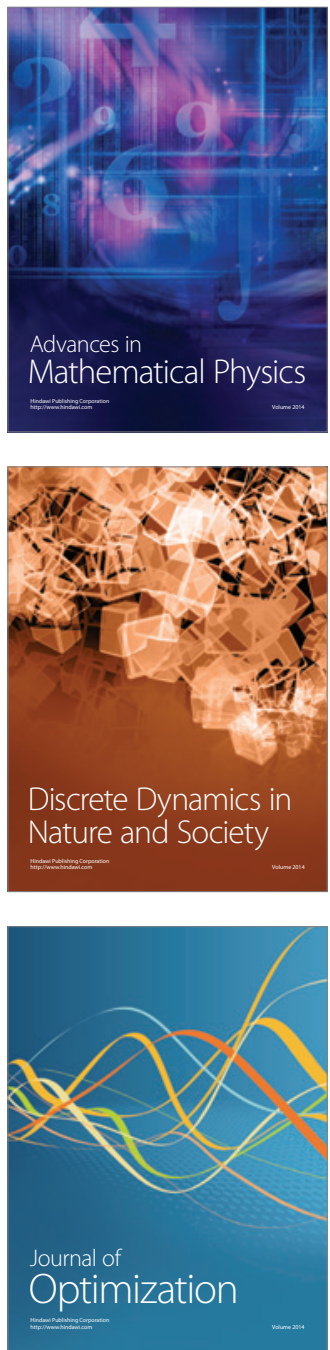